\title{
Critically III Medical Patient Outcomes after Discharge from Intensive Care - the prospective longitudinal 'CIMPOD' study of Malta's national ICU survivors
}

\author{
Andrew Grech, Carmel Abela \\ Department of Anaesthesia, Intensive Care \& Pain Medicine, Mater Dei Hospital, Malta
}

\section{Background}

Intensive care survivors represent a product of considerable resource expenditure, both economic and intangible. While survival of a period of critical illness is invariably a marker of some degree of successful short term outcome, the literature base for general and local intensive care unit (ICU) practice in particular exhibits a lacuna for awareness of patient outcomes after ICU discharge.

\section{Aims}

The primary aim involves description of the prevalence of post-ICU outcomes related to mortality, urgent readmission events, functional status and emotional outcomes in national ICU practice. Secondary objectives include identification of potential predictors of outcomes for use in future risk stratification exercises.

\begin{tabular}{|l|l|}
\hline 76 & - Discharged from ICU care \\
\hline 62 & - Duration of ICU stay $>24$ hours \\
\hline 51 & - Age $>16$ years \\
\hline 55 & - Non-foreign nationals \\
\hline
\end{tabular}

Fig. I Eligibility criteria for study participation and recruitment

\section{Methods}

Consecutive ICU survivors aged $>16$ years were eligible for recruitment over a four week period having spent $>24$ hours in our national ICU facility. Age, gender and duration of ICU stay were recorded and retrospective acute physiology and chronic health evaluation (APACHE-II) illness severity scores calculated for the admission. Fifty five patients were followed up over the study period, with interval follow up performed at one month, three months and ultimately six months after ICU discharge to assess outcome measures as above. Health related quality of life was evaluated via use of the EuroQOL EQ-5D-3L instrument, while emotional outcomes were screened using the Hospital Anxiety and Depression Scale.

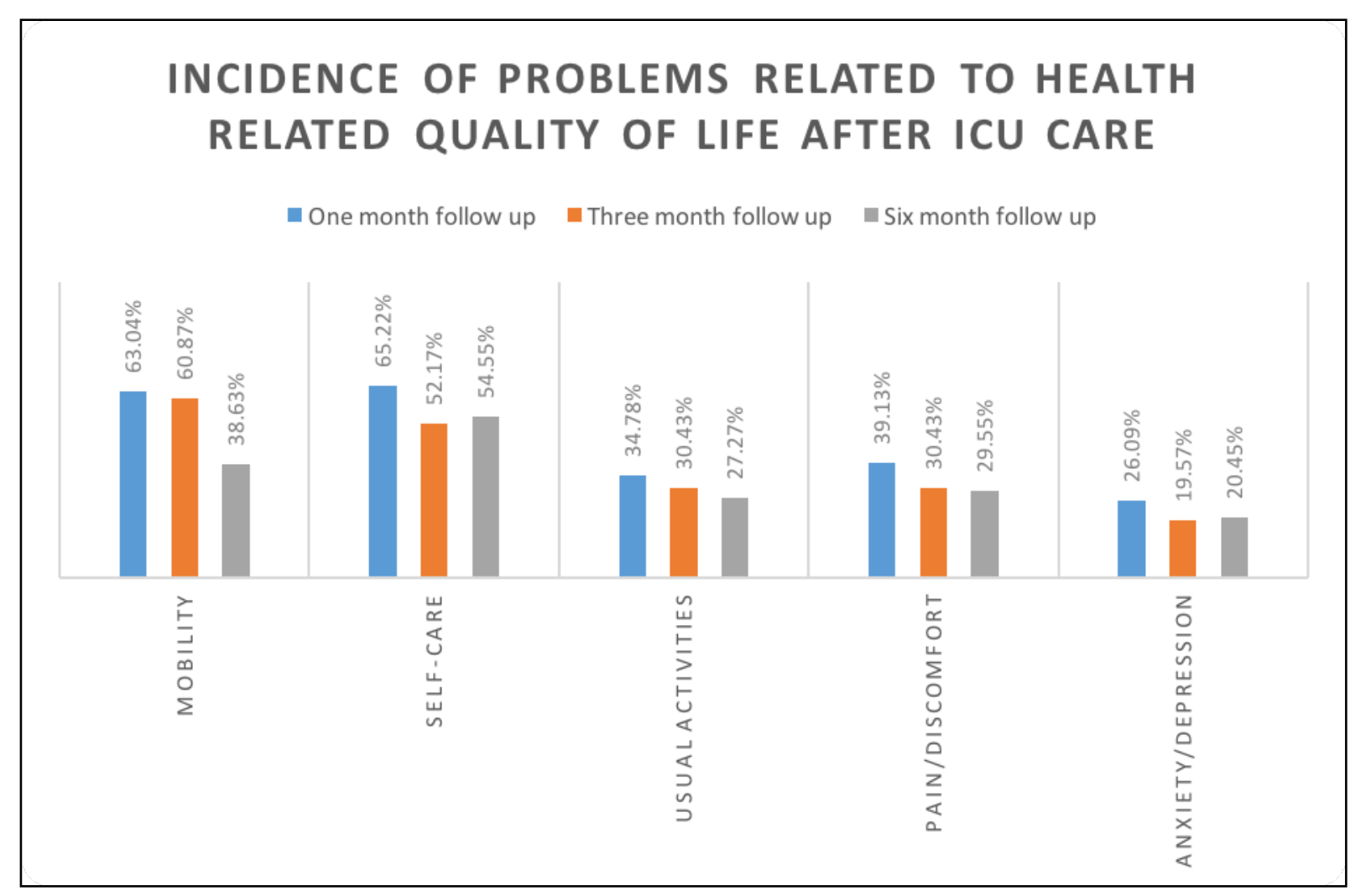

Fig. 2 Health related quality of life after ICU care as per EQ-5D-3L scale

\section{Results}

Mortality was noted in $16.4 \%$ at one month after ICU discharge, reaching $20 \%$ at six months. A majority of participants (60\%) did not exhibit any urgent readmission events to state hospital services by the study's conclusion.

Mean overall health status perception on EQ-5D-3L visual analogue scale (VAS) improved from $58.59( \pm 15.12)$ at one month to 68.07 $( \pm 16.18)$ at six months.

Anxiety and depression scores showed positive correlation throughout, with $22.7 \%$ and $11.36 \%$ of participants ultimately demonstrating positive screening values for anxiety and depression potential respectively. Several statistically significant logistic regression models were interpreted, implicating age as a potential predictor of perceived health status and similarly gender for anxiety screening scores.

Length of ICU stay was implicated as an independent factor associated with anxiety, depression and health status perception at one month after ICU illness.

APACHE-II scores calculated for this study demonstrated significant potential as predictors of tangible post-ICU outcomes linked to mortality and readmission events across the six months following critical illness.

\begin{tabular}{|c|c|}
\hline Independent factor & Outcome \\
\hline Patient age & 3 month VAS score \\
\hline Gender & 6 month VAS score \\
\hline & I month HADS anxiety \\
\hline & 3 month HADS anxiety \\
\hline APACHE-II admission score & month HADS anxiety \\
\hline & I month mortality \\
\hline & 6 month mortality \\
\hline & I month readmission status \\
\hline & 3 month readmission status \\
\hline & 6 month readmission status \\
\hline Duration of ICU stay & I month VAS score \\
\hline & 3 month VAS score \\
\hline & I month HADS anxiety \\
\hline & I month HADS anxiety \\
\hline & \\
\hline
\end{tabular}

Tab. I Statistically significant $(p<0.05)$ potential outcome predictors

\section{Conclusions}

While limited by its size, the CIMPOD study into post-ICU outcomes represents one of the first of its kind in Maltese practice. Mortality and readmission after leaving ICU might reflect a portion of less sick patients entering ICU in the first instance due to absence of a local high dependency unit, yet outcomes in the same population nevertheless show potential for future research.

Relationship modelling in this preliminary study appears to indicate scope for risk stratification practices in local ICU medicine, with APACHE-II scoring demonstrating particular potential. Further study of greater numbers of patients using similar research protocols is suggested for future focus, while considerations for introduction of sickness severity scoring in our ICU environment are recommended. 